\title{
Optical photometric and spectral study of the new FU Orionis object V2493 Cygni (HBC 722)^ (Research Note)
}

\author{
E. H. Semkov ${ }^{1}$, S. P. Peneva ${ }^{1}$, U. Munari² ${ }^{2}$, M. K. Tsvetkov ${ }^{1}$, R. Jurdana-Šepić ${ }^{3}$, E. de Miguel $^{4}$, R. D. Schwartz ${ }^{5}$, \\ D. P. Dimitrov ${ }^{1}$, D. P. Kjurkchieva ${ }^{6}$, and V. S. Radeva ${ }^{6,7}$

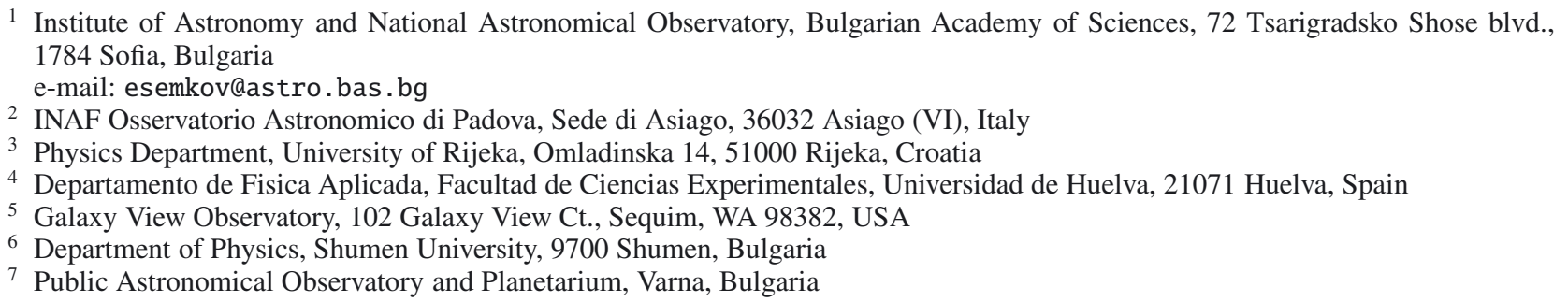

Received 29 February 2012 / Accepted 24 April 2012

\section{ABSTRACT}

\begin{abstract}
Aims. We present new results from optical photometric and spectroscopic observations of the eruptive pre-main sequence star V2493 Cyg (HBC 722). The object has continued to undergo significant brightness variations over the past few months and is an ideal target for follow-up observations.

Methods. We carried out CCD BVRI photometric observations in the field of V2493 Cyg ("Gulf of Mexico") from August 1994 to April 2012, i.e. at the pre-outburst states and during the phases of the outburst. We acquired high, medium, and low resolution spectroscopy of V2493 Cyg during the outburst. To study the pre-outburst variability of the target and construct its historical light curve, we searched for archival observations in photographic plate collections. Both CCD and photographic observations were analyzed using 15 comparison stars in the field of V2493 Cyg.

Results. The pre-outburst photographic and CCD photometric observations of V2493 Cyg show low-amplitude light variations typical of T Tauri stars. The recent photometric data show a slow light decrease from October 2010 to June 2011 followed by an increase in brightness that continued until early 2012. The spectral observations of V2493 Cyg are typical of FU Orionis stars absorption spectra with strong P Cyg profiles of $\mathrm{H} \alpha$ and $\mathrm{Na}$ I D lines. On the basis of photometric monitoring performed over the past two years, the spectral properties at the maximal light, as well as the shape of long-term light curves, we confirm that the observed outburst of V2493 Cyg is of FU Orionis type.
\end{abstract}

Key words. stars: pre-main sequence - stars: variables: T Tauri, Herbig Ae/Be - stars: individual: V2493 Cygni

\section{Introduction}

Studies of the photometric and spectroscopic variability of premain sequence (PMS) stars are very important to improving our understanding of the early stages of stellar evolution. One of the most remarkable PMS phenomena associated with a significant increase in brightness is the FU Orionis (FUor) outburst. This corresponds to young eruptive stars as originally classified by Herbig (1977) following the discovery of the FUor objects V1057 Cyg and V1515 Cyg. Several more objects have since been assigned to this class of young variables (see Reipurth \& Aspin 2010, and references therein). All known FUors share the same defining characteristics: a $\Delta V \approx 4-6$ mag outburst amplitude, association with reflection nebulae, location in starforming regions, an F-G supergiant spectrum during outbursts, a strong LiI $6707 \AA$ line in absorption, and $\mathrm{CO}$ bands in near-infrared spectra (Herbig 1977; Reipurth \& Aspin 2010). An important feature of FUors is the massive supersonic wind

* Tables 1 and 3 are only available at the CDS via anonymous ftp to cdsarc.u-strasbg.fr $(130.79 .128 .5)$ or via

http://cdsarc.u-strasbg.fr/viz-bin/qcat?J/A+A/542/A43 observed as a P Cyg profile most commonly for both $\mathrm{H} \alpha$ and $\mathrm{Na} I \mathrm{D}$ lines. A typical outburst of FUor objects can last for several decades, and the rise time is shorter than that of the decline.

Herbig (1989) also defined another class of young eruptive stars characterized by more than one outburst in the optical light, and referred to them as EXors after the prototype of this class, the PMS star EX Lupi. These EXor objects undergo frequent (every few years or decades), irregular, and relatively brief (a few weeks to a few months or one year) outbursts with amplitudes $\Delta V \approx 2-5$. During these events, the cool spectrum of the quiescence is veiled and strong emission lines from single ionized metals appear with reversed P-Cyg absorption components (Herbig 2007; Aspin et al. 2010). Both types of eruptive stars, FUors and EXors, seem to be related to the low-mass PMS objects (T Tauri stars), which have massive circumstellar disks. Their outbursts are generally attributed to the infall of material from the circumstellar disk to the central star (Hartmann \& Kenyon 1996).

The large-amplitude outburst of V2493 Cyg (HBC 722) in the summer of 2010 (Semkov \& Peneva 2010a; Miller et al. 2011) generated considerable interest and was studied across a 
wide spectral range. Before the outburst, the target had not been registered as a variable and there were no published data from its optical photometric studies. Follow-up photometric and spectroscopic observations by Semkov \& Peneva (2010b), Munari et al. (2010), Leoni et al. (2010), Semkov et al. (2010), and Kóspál et al. (2011) recorded an ongoing light increase in both the optical and infrared, as well as significant changes in the spectrum of V2493 Cyg. Miller et al. (2011) reported on their infrared photometry and spectroscopy, as well as low and high resolution optical spectroscopy of the target. They confirmed its FUor nature and noted that it is the first FUor associated with a previously known young stellar object. The authors also commented on the spectral classification of V2493 Cyg appearing to progress from G-type at blue optical wavelengths to K-type toward the red.

Results from a high resolution, optical, spectroscopic campaign of V2493 Cyg during November-December 2010 are given by Lee et al. (2011). Optical and near-infrared JHK photometry of V2493 Cyg was presented by Lorenzetti et al. (2011), Kóspál et al. (2011), and Lorenzetti et al. (2012). The preoutburst spectral energy distribution (SED) of V2493 Cyg is discussed in the papers of Miller et al. (2011) and Kóspál et al. (2011). These authors concluded that V2493 Cyg had been a Class II young stellar object before the eruption, which is an object type most often associated with classical T Tauri stars. The pre-outburst bolometric luminosity of the object was estimated as $0.85 L_{\odot}$ (Kóspál et al. 2011) while during the maximum light this luminosity rose to $\sim 12 L_{\odot}$ (Miller et al. 2011).

We also highlight the case of V2493 Cyg because it is the first (and so far the only) FUor object detected in X-rays during outburst. The detection was obtained by Pooley \& Green (2010) observing in the $0.2-10 \mathrm{keV}$ band with the Swift X-ray satellite. Green et al. (2011) analyzed the submillimeter CO emission surrounding V2493 Cyg using images and spectroscopy from the Herschel Space Observatory and the Caltech Submillimeter Observatory. The authors concluded that V2493 Cyg "did not show evidence for a circumstellar envelope or shocked gas, and appeared to erupt from a disk-like state, similar to FU Orionis itself".

\section{Observations}

\subsection{Photometric CCD observations}

The CCD photometric observations of V2493 Cyg were performed with the 2-m RCC, the 50/70-cm Schmidt, and the $60-\mathrm{cm}$ Cassegrain telescopes of the National Astronomical Observatory Rozhen (Bulgaria), the 1.3-m RC telescope of the Skinakas Observatory $^{1}$ of the Institute of Astronomy, University of Crete (Greece), the 42-cm telescope of the Galaxy View Observatory, Sequim (USA), and both the $25-\mathrm{cm}$ Newtonian and the $29-\mathrm{cm}$ Schmidt Cassegrain telescopes of the Observatorio Astronómico del CIECEM, Universidad de Huelva (Spain). All frames were exposed through a set of standard Johnson-Cousins filters.

Our first CCD observations of the field around V2493 Cyg were performed with the Rozhen 2-m RCC telescope and SBIG ST-6 camera in 1994-1996. The subsequent set of observations with this telescope were made with the CCD cameras Photometrics AT-200 and VersArray 1300B. The observations with the 50/70-cm Schmidt telescope were performed with the cameras: SBIG ST-8 in 2000-2007, SBIG STL11000 from 2008

\footnotetext{
1 Skinakas Observatory is a collaborative project of the University of Crete, the Foundation for Research and Technology - Hellas, and the Max-Planck-Institut für Extraterrestrische Physik.
}

to Apr. 16, 2009, and FLI PL 16803 from July 2009 to the present. Observations with the Skinakas 1.3-m RC telescope were performed with the Potometrics $\mathrm{CH}-360$ CCD camera from Jun. 2000 to Jul. 3, 2007 and with the ANDOR DZ 436 CCD camera from Jul. 23, 2007 to the present. After registration of the outburst, observations of V2493 Cyg were made with: the 60-cm Cassegrain telescopes of the Rozhen Observatory with the FLI PL 9000 CCD camera, the 42-cm telescope of the Galaxy View Observatory with the SBIG ST-8XME CCD camera, and the 25-cm Newtonian and the 29-cm Schmidt Cassegrain telescopes of the Observatorio del CIECEM with a QSI-516ws CCD camera.

All the data were analyzed using the same aperture, which was chosen as $4^{\prime \prime}$ in radius (while the background annulus was from $13^{\prime \prime}$ to $19^{\prime \prime}$ ) in order to minimize the light from the surrounding nebula and avoid contamination from adjacent stars. As references, we used the BVRI comparison sequence of fifteen stars in the field around V2493 Cyg published in Semkov et al. (2010). In this way we provided a maximum consistency of the photometric results obtained at different telescopes and CCD cameras.

The results of our photometric CCD observations of V2493 Cyg are summarized in Table 1. The columns provide the date and Julian date (JD) of observation, IRVB magnitudes of V2493 Cyg, the telescope and CCD camera used. The typical errors in the reported magnitudes before the outburst are $0.01-0.02$ for $I$ and $R$-band data, $0.02-0.06$ for $V$, and 0 . $03-0 .{ }^{\mathrm{m}} 10$ for $B$-band. The large increase in the brightness associated with the outburst contributed to a significant reduction in the error in the CCD photometry of V2493 Cyg obtained during the ongoing bright state. Since August 2010 the typical errors in the reported magnitudes are $0 \mathrm{~m} 01$ for $I$ and $R$-band data, 0 . $01-0.02$ for $V$, and $0.02-0$. 05 for $B$-band.

\subsection{Spectral observations}

Medium-resolution, single-dispersion spectroscopy of V2493 Cyg was obtained on Nov. 21, 2011 with the AFOSC spectrograph+imager mounted on the Asiago 1.82-m reflector and equipped with a $1720 \mathrm{ln} / \mathrm{mm}$ volume phase holographic grism and a 2.1 arcsec slit oriented east-west (E-W). Medium and low resolution, single dispersion spectroscopy of V2493 Cyg was obtained on Nov. 30 and Dec. 1, 2011, with the Asiago 1.22-m reflector equipped with $1200 \mathrm{ln} / \mathrm{mm}$ and $300 \mathrm{ln} / \mathrm{mm}$ gratings. The slit was widened to 2.0 arcsec and oriented E-W. Absolute flux calibration was obtained by observing spectrophotometric standard stars both just before and soon after the observations of V2493 Cyg and at similar airmasses. Finally, a $2 \times 2$ binned echelle spectrum was obtained on Jan. 13, 2012 with the REOSC echelle spectrograph mounted on the $1.82-\mathrm{m}$ telescope, with a 2.2 arcsec wide slit oriented E-W. All data reduction was performed within IRAF, with Table 2 providing a log of spectral observations.

\subsection{Archival photographic observations}

The region of NGC 7000/IC 5070 is one of the most well studied areas of star formation in our galaxy. The field is rich in young stellar objects with different masses, $\mathrm{H} \alpha$ emission stars, Herbig-Haro objects, flare stars from UV Ceti type, and other irregular variable young objects (Reipurth \& Schneider 2008; Armond et al. 2011). On the other hand, the bright North America and Pelican nebulae have been continuously attracting 
Table 2. Journal of spectroscopic observations.

\begin{tabular}{lcclcc}
\hline \hline Date & UT & $\begin{array}{c}\text { Expt } \\
(\mathrm{s})\end{array}$ & & $\begin{array}{c}\lambda \text { range } \\
(\AA)\end{array}$ & Tel. \\
\hline $2011-11-21$ & $20: 08$ & 3600 & disp. $0.72 \AA /$ pix & $6350-7080$ & $1.82 \mathrm{~m}+\mathrm{AFOSC}$ \\
$2011-11-30$ & $20: 28$ & 1800 & disp. $0.61 \AA /$ pix & $5680-6910$ & $1.22 \mathrm{~m}+\mathrm{B} \& \mathrm{C}$ \\
$2011-12-01$ & $19: 01$ & 7200 & disp. 2.31 $\mathrm{\AA} / \mathrm{pix}$ & $3700-7550$ & $1.22 \mathrm{~m}+\mathrm{B} \& \mathrm{C}$ \\
$2012-01-13$ & $17: 19$ & 3600 & res. pow. 12000 & $4400-7335$ & $1.82 \mathrm{~m}+$ echelle \\
\hline
\end{tabular}

the interest of astrophotographers and researchers worldwide, and as a consequence the plate archives of several observatories preserve abundant collections of plates exposed over several decades on this region of the sky. The collection and analysis of all these observations is very valuable for the study of the longtime variability of V2493 Cyg but it requires a very long and laborious amount of work.

In this paper, we present the result of exploring the whole photographic plate stack preserved at two observatories, the Asiago Observatory (Italy), and the National Astronomical Observatory Rozhen (Bulgaria). The digitized plates from the Palomar Schmidt telescope, available via the website of the Space Telescope Science Institute, are also used. Photographic observations in the field of V2493 Cyg were performed with the $67 / 92 \mathrm{~cm}$ and the 40/50 $\mathrm{cm}$ Schmidt telescopes at the Asiago Observatory and with the 2-m RCC telescope and the 50/70 cm Schmidt telescope at the Rozhen Observatory. In addition, we used several scanned plates from the 100/130 cm Schmidt telescope of the Byurakan Astrophysical Observatory (Armenia), the $30 \mathrm{~cm}$ Astrograph of the Hoher List Observatory (Germany), and the historic first Schmidt-type telescope $(36 / 44 \mathrm{~cm})$ mounted on the Hamburg-Bergedorf Observatory (Germany). These plates are available from the Sofia Sky Archive Center the Wide-field Plate Database ${ }^{2}$. The plates from Rozhen, Byurakan, Hoher List, and Hamburg-Bergedorf were scanned with Epson Expression 1640 XL/10000XL scanners, which have 1600-2400 dpi resolution and a corresponding pixel size from $16 \times 16 \mu \mathrm{m}$ to $10 \times 10 \mu \mathrm{m}$. Aperture photometry of the digitized plates was performed with DAOPHOT routines using the same aperture radius and the background annulus as for the CCD photometry. The BVRI comparison sequence reported in Semkov et al. (2010) was used as a reference. Figure 1 illustrates the quality of the photographic plates used, regardless of their age.

The plates from Asiago Schmidt telescope were inspected visually using a high quality Carl Zeiss microscope, which offered a variety of magnifications (Munari et al. 2001). The magnitude was then derived by comparing the variable with the stars in the same photometric sequence adopted for the reduction of the CCD observations. When "a" and " $b$ " are two stars in the sequence and $V$ is the variable, we can estimate the brightness of the variable on photographic plates inspected visually with a microscope, where "a" is slightly brighter than $V$ and "b" slightly fainter. The difference in brightness between " $a$ " and " $b$ " as perceived by the eye is divided into ten steps, and the difference in magnitude between $V$ and the two stars is counted in terms of these steps, as $\mathrm{n} 1$ and $\mathrm{n} 2$, conventionally expressed as "a $\mathrm{n} 1 V \mathrm{n} 2 \mathrm{~b}$ ", with $\mathrm{n} 1+\mathrm{n} 2=10$. The magnitude of the variable $V$ is then obtained directly from the simple arithmetic proportion

$\operatorname{mag}(V)=\{[\operatorname{mag}(\mathrm{b})-\operatorname{mag}(\mathrm{a})] / 10\} * \mathrm{n} 1+\operatorname{mag}(\mathrm{a})$.

\footnotetext{
2 wWW.wfpdb.org
}

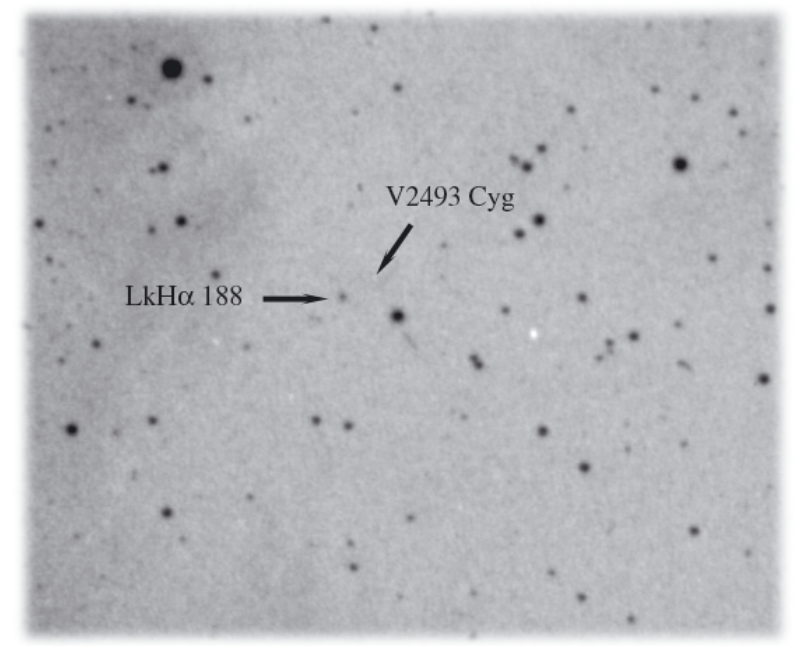

Fig. 1. A copy of the photographic plate obtained in the region of NGC 7000 with the first Schmidt telescope in the Hamburg-Bergedorf Observatory on Dec. 181932 (exposure: two hours, observer: Bernhard Schmidt). The brightest $\mathrm{H} \alpha$ emission star in the field $\mathrm{LkH} \alpha 188$ and the position of V2493 Cyg are marked by arrows. The plate limit is estimated at $17.3(\mathrm{pg})$. Three of our standard stars $(\mathrm{A}-\mathrm{C})$ are visible at the bottom-left of $\mathrm{LkH} \alpha 188$.

If more than one such pair were available, more estimates would be derived and weighted for the "ab", "cd" etc. mag interval. Typical estimated errors are on the order of $0.10 \mathrm{mag}$.

The results of the measured magnitudes of V2493 Cyg from the archival photographic plates are given in Table 3 . The columns provide the name of the observatory, the plate number, the date and JD of observation, photographic emulsions and filters used, the magnitude estimated or plate limit, and the corresponding errors. Moreover, V2493 Cyg is invisible $\left(\mathrm{pg}<17^{\mathrm{m}}\right)$ in the photographic plate obtained by Max Wolf on 1907 Aug. 07 with the 72-cm Waltz-Reflector in the Königsstuhl Observatory, Heidelberg (Germany) (Wenzel 2011).

\section{Results and discussion}

Photometric data presented in this paper show the usefulness of systematically monitoring the star forming regions. These data can be used to detect new FUor or EXor events and to determine the type of the outburst. The BVRI light curves of V2493 Cyg are plotted in Fig. 1. The filled diamonds represent the CCD observations from the present paper, the filled circles observations from the 48 inch Samuel Oschin telescope at Palomar Observatory (Miller et al. 2011), the open diamonds photographic data from the Asiago Schmidt telescopes, the open squares photographic data from the Palomar Schmidt telescope, the filled squares photographic data from the Byurakan Schmidt telescope and the open circles photographic data from the Rozhen 2-m RCC telescope. The optical photometric data 


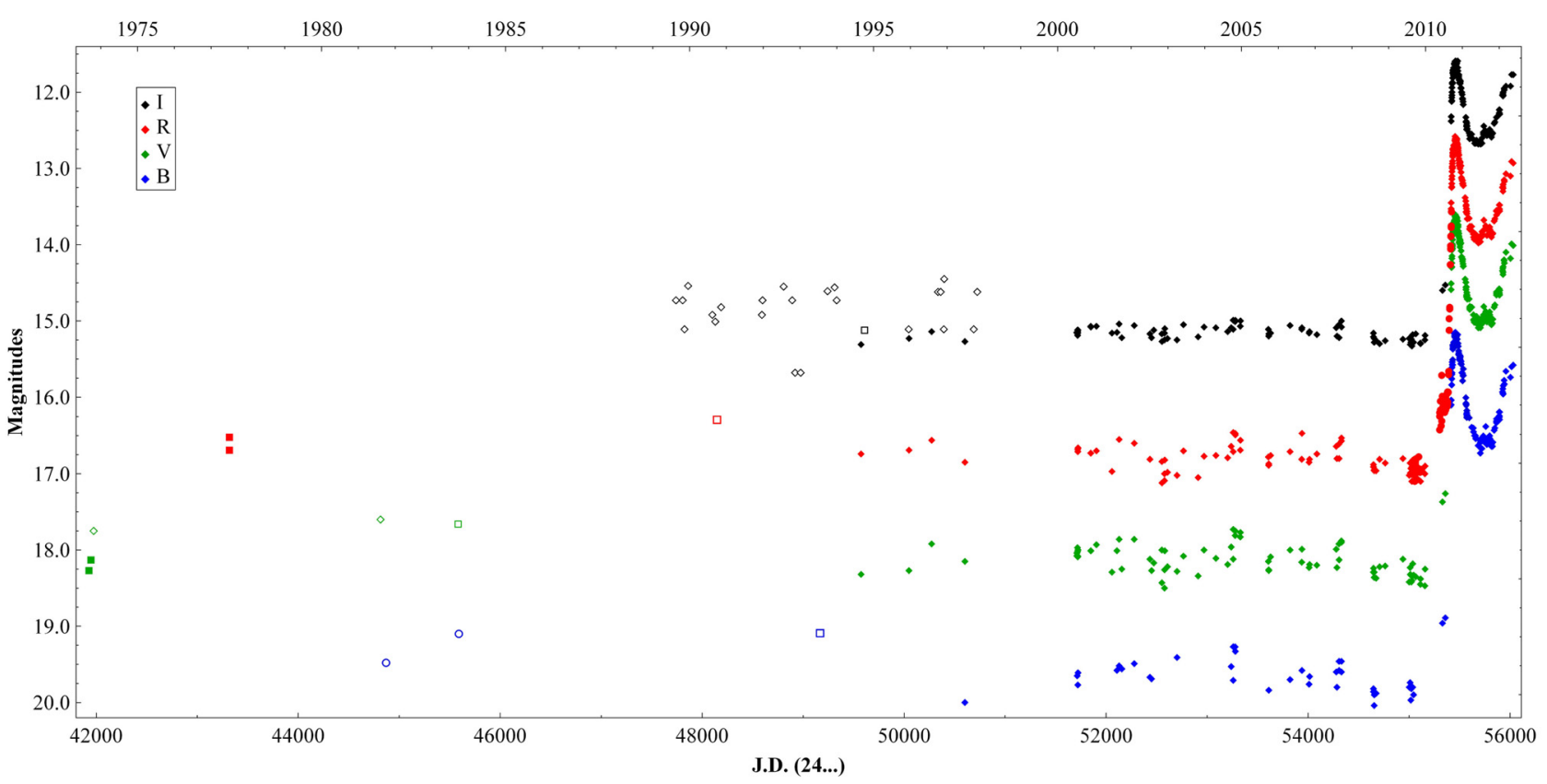

Fig. 2. Historical BVRI light curves of V2493 Cyg for the period September 1973-April 2012.

published by other authors (Kóspál et al. 2011; Lorenzetti et al. 2012) show the same changes in brightness during the outburst, which are perfectly matched with the light curves shown in Fig. 1.

The historical light curves of V 2493 Cyg allows us to conclude that its photometric behavior is similar to that of a classical $\mathrm{T}$ Tauri star. The same conclusion was made by Lorenzetti et al. (2011) based on a comparison of infrared photometry from Cohen \& Kuhi (1979) and from the 2MASS catalog. Other large-amplitude eruptions have not yet been registered in our long-term photometric study, but due to the sparse and random photometry available at this stage, the occurrence of these short events cannot be ruled out. After reaching its maximum brightness in October 2010 (Semkov et al. 2010), the brightness of V2493 Cyg declined slowly, having weakened by $1.45(V)$ by the spring/early summer of 2011. During the summer of 2011, V2493 Cyg underwent only minor brightness variations around the mean level that was brighter than the quiescence level by $3.3(V)$. From October 2011, another light increase occurred and the star became brighter by $1.1 \mathrm{~m}(V)$ until April 2012. Consequently, the outburst of V2493 Cyg continued for approximately two years.

Figure 3 represents the change in position of V2493 Cyg during the outburst on the color-color diagram $V-R / R-I$. The location of main-sequence dwarfs (blue line) according to Johnson (1966) and the interstellar reddening vector for the region $A_{V}=3$. 4 (black line) according to Cohen \& Kuhi (1979) are shown. Data for the main-sequence stars are recalculated to correspond to the Johnson's to Cousins system using the corresponding equations in Moro \& Munari (2000). Figure 3 shows that V2493 Cyg becomes appreciably bluer when its brightness increases during the outburst.

The spectral observations obtained during the past few months show substantial changes in the profile of $\mathrm{H} \alpha$ line. Figure 4 presents a comparison between the low-resolution spectra of V2493 Cyg obtained at the beginning of outburst (September 2010) and during the recent increase in brightness (December 2011). In the first spectrum, the $\mathrm{H} \alpha$ line is seen in emission, which is confirmed by the low-resolution spectroscopy

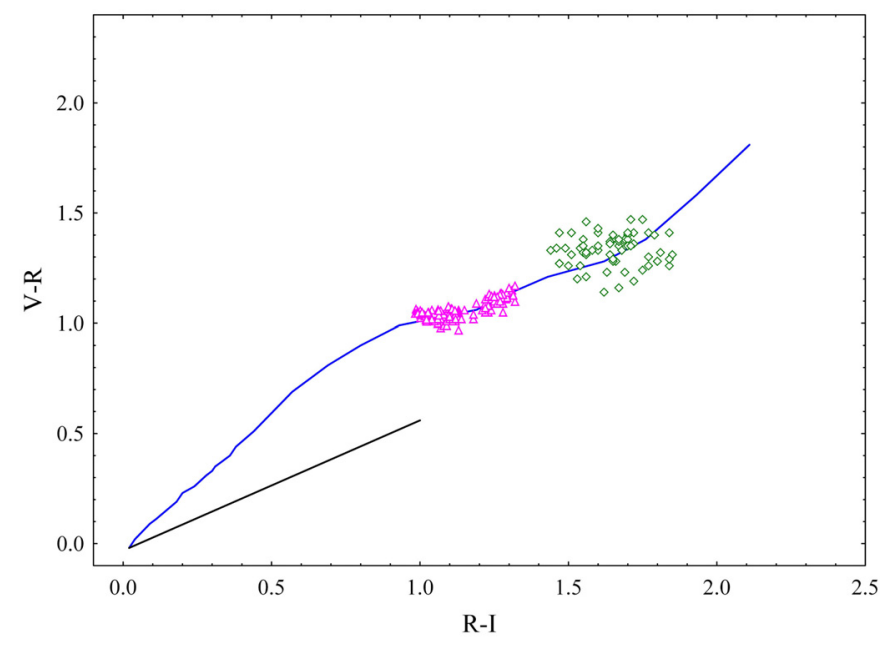

Fig. 3. $V-R$ vs. $R-I$ color-color diagram for V2493 Cyg before (open diamonds) and during the outburst (open triangles). The solid blue line is the locus of main-sequence dwarf stars. The straight black line is the reddening vector for $A_{V}=3$. 4 .

of Miller et al. (2011). The second spectrum already clearly shows the strong P Cyg shape of the $\mathrm{H} \alpha$ line. A significant change in the SED of V2493 Cyg relative to both spectra is observed, as also confirmed by the photometric data (Table 1). During the second rise in brightness, the star is significantly bluer than during the first.

From the high-resolution spectroscopy, the $\mathrm{P}$ Cyg profile of the $\mathrm{H} \alpha$ line was detectable at the beginning of the outburst (Miller et al. 2011; Lee et al. 2011). The deep and highvelocity blueshifted absorption is interpreted as evidence of a strong outflow driven by the FUor object. According to Miller et al. (2011), the blueshifted absorption of $\mathrm{H} \alpha$ line extends up to $\sim-200 \mathrm{~km} \mathrm{~s}^{-1}$ on September 2010. Our most recent high and medium resolution spectroscopy (Table 2) indicate that there has been a significant increase in the velocity of the absorption component of $\mathrm{H} \alpha$ and $\mathrm{Na} \mathrm{I} \mathrm{D} \mathrm{lines} \mathrm{(Fig.} \mathrm{5).} \mathrm{The} \mathrm{terminal}$ 


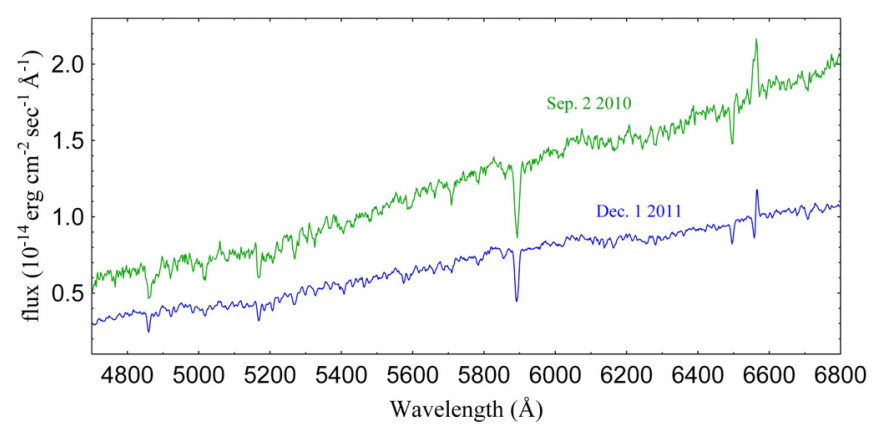

Fig. 4. Low-resolution spectra of V2493 Cyg obtained on Sep. 2, 2010 (the 60-cm telescope of Schiaparelli Observatory in Varese) and on Dec. 1, 2011 (1.22-m telescope of Asiago Observatory).

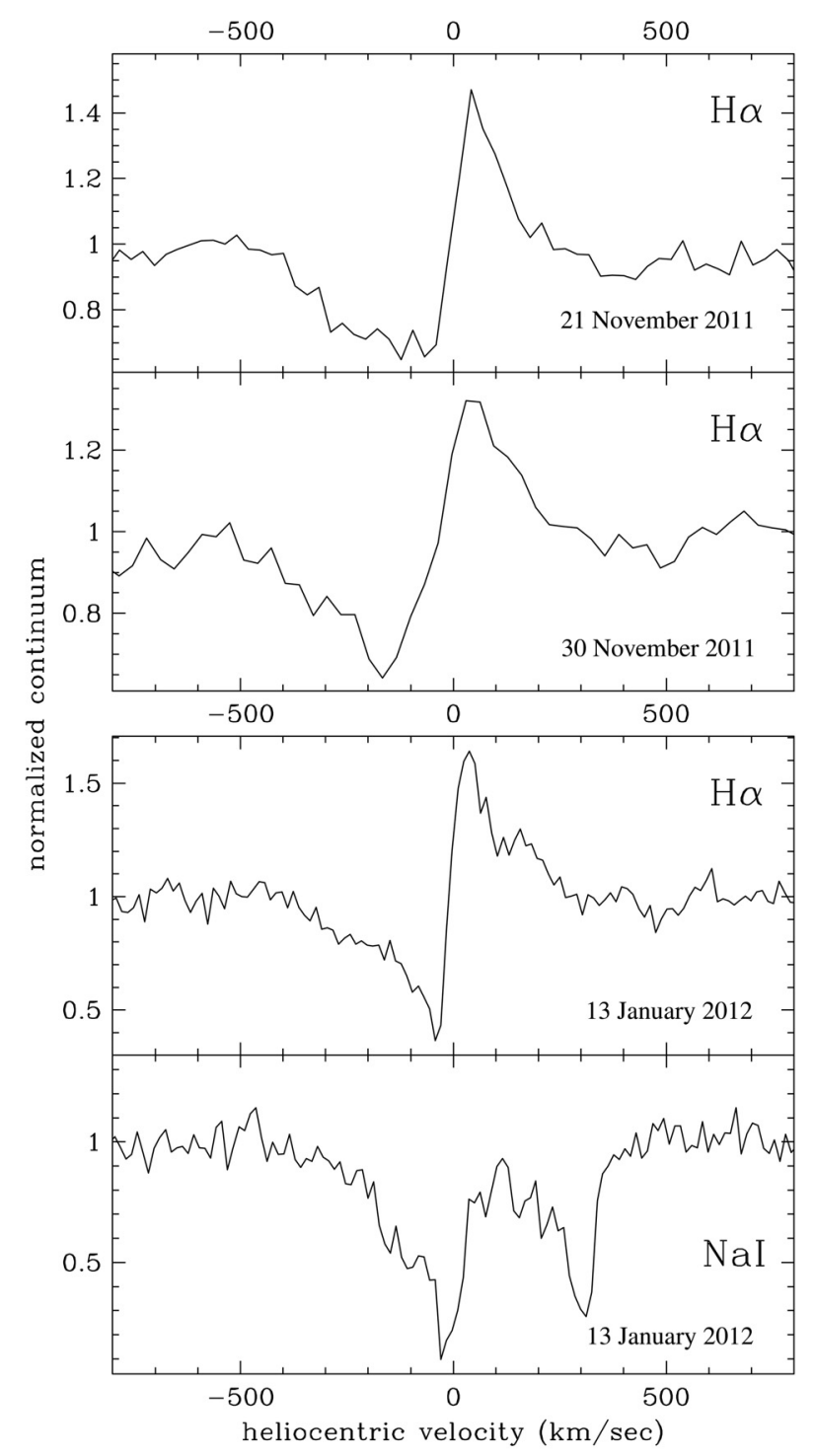

Fig. 5. The profiles of $\mathrm{H} \alpha$ and $\mathrm{Na}$ I D lines extracted from the three spectra of V2493 Cyg obtained during the second increase in brightness (cf. Table 2).

velocity of the wind during the two months covered by our high resolution monitoring remained stable at about $-500 \mathrm{~km} \mathrm{~s}^{-1}$, while profound changes were observed in the shape of the absorption component.
The interpretation of the profile changes of the absorption component is complicated by the uncertainties in the location and the precise mechanism responsible for the formation of the line, which may be a spherically symmetric wind, blowing from either the stellar surface or a circumstellar disk, that is radiatively accelerated, etc. The changes observed in the profiles of Fig. 5 could be for example accounted for by a variation in the optical depth in the line. In this framework, the profile for Nov. 21, 2011 would correspond to an optical depth larger than that measured on Jan. 13, 2012, with the profile for Nov. 30, 2011 being characterized by an in-between value of the optical depth. There has been no noticeable correlation between the variations in the optical depth of $\mathrm{H} \alpha$ line and the photometric properties during recent months. Such a correlation is also not found in other classical FUors that appear to have a highly variable stellar wind and no significant changes the brightness (Herbig et al. 2003; Herbig 2009).

To classify the absorption spectrum of V2493 Cyg, we compared our spectra with the Asiago atlas of MKK spectral types observed with exactly the same instrumental configuration of V2493 Cyg (Munari, in prep.). We took the absolutely fluxed spectra of V2493 Cyg and the MKK atlas and continuum normalized them using the same function (a Legendre polynomial of fifth order limited to the range of wavelength covered in Fig. 6 which correspond to those recommended for the classification within the MKK system). As a first classification pass, we applied a simple $\chi^{2}$ matching to determine the area of the Herzsprung-Russell diagram on which our deeper analysis was focused. The match found by the $\chi^{2}$ is not perfect, since the stellar spectra originates in stationary atmospheres where a three-dimensional treatment is generally unnecessary, while the absorption lines in V2493 Cyg instead form in a moving medium, the wind. We then proceeded to refine the classification by using an eye inspection of the spectra, and found that the closest (even though imperfect) match was for a G3I type star. Figure 6 shows how the properties of the absorption spectrum of V2493 Cyg are in-between those of supergiants of the G0 and G5 types.

All published studies of V2493 Cyg eruption tried to determine whether it is either a FUor or EXor type star. Most authors (Semkov et al. 2010; Miller et al. 2011; Green et al. 2011; Lee et al. 2011; Pooley \& Green 2010; Reipurth et al. 2012) classified V2493 Cyg as a typical FUor, but others (Kóspál et al. 2011; Leoni et al. 2010; Lorenzetti et al. 2012) considered the observed outburst to have instead a EXor type. Both types of PMS eruptive stars have been classified in terms of their wide range of available photometric and spectral properties, but their outbursts are thought to have the same cause - an enhanced accretion rate. The observed eruption of V1647 Ori appears to have characteristics similar to those of FUor and EXor, and it is assumed to be inbetween the two types. According to Aspin (2011), the viewing inclination angle of the star/disk system can play a significant role in the observed spectral features. Therefore, it is assumed that the two types of outbursts may be much closer in nature.

Unlike the classical FUor stars, V2493 Cyg has a relatively low outburst luminosity $\sim 12 L_{\odot}$ (Miller et al. 2011) and low accretion rate $\sim 10^{-6} M_{\odot} /$ yr (Kóspál et al. 2011). Most of the FUor objects have luminosities on the order of $100 L_{\odot}$ during the maximum. The luminosity of V2493 Cyg is at the faint end of the luminosity range of FUor outbursts but still comparable to those of some FUor objects such as L1551 IRS5 and HH381 IRS (Reipurth \& Aspin 2010).

From all objects associated with the group of FUors, only three (FU Ori, V 1057 Cyg, and V 1515 Cyg) have detailed 


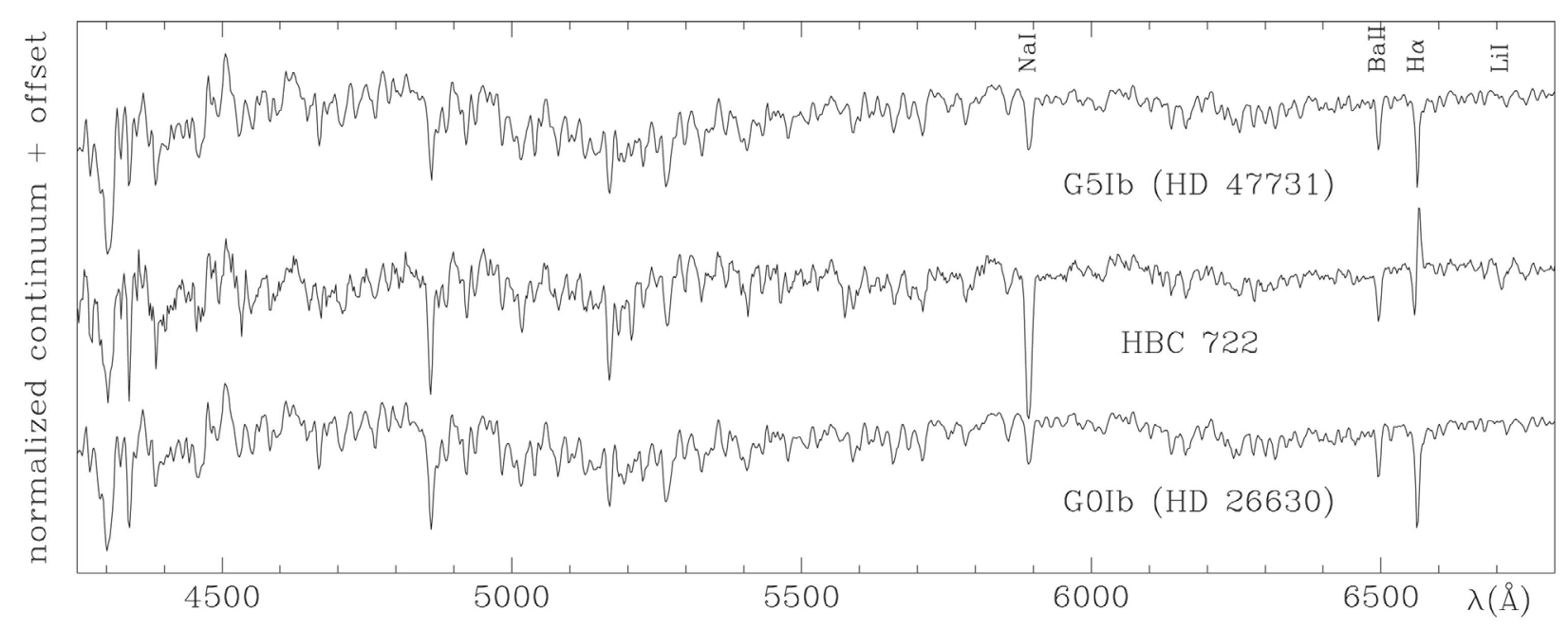

Fig. 6. High-resolution spectrum of V2493 Cyg (HBC 722) is compared with spectra of the G supergiant stars HD 47731 and HD 26630 (from Asiago spectral database).

photometric observations taken during the outburst and during the set of brightness (Clarke et al. 2005). For some years, we have made efforts to construct the historical light curves of several other FUor and FUor-like objects such as: V1735 Cyg (Peneva et al. 2009), Parsamian 21 (Semkov \& Peneva 2010c), V733 Cep (Peneva et al. 2010), and V582 Aur (Semkov et al. 2011). Our results suggest that each object of this type has a characteristic long-term light curve, which distinguishes it from other objects. The shape of the observed light curves of FUors may vary considerably in the time of rise, the rate of decrease in brightness, the time spent at maximum brightness, and the light variability during the set in brightness.

The light curve of V2493 Cyg from all available photometric observations is also somewhat unique. The rate of increase in the brightness (the fastest ever recorded) was followed by a very rapid fall in brightness. But the most remarkable feature of the light curve of V2493 Cyg is the repeated rise in brightness in the past few months. At present, it is impossible to predict how long the rise in brightness will continue and which maximal stellar magnitude will be exceeded.

\section{Concusions}

On the basis of our photometric monitoring over the past two years and the spectral properties at the maximal light (a G3I supergiant spectrum with strong $\mathrm{P}$ Cyg profiles of $\mathrm{H} \alpha$ and $\mathrm{Na}$ I D lines), we have confirmed that the observed outburst of V2493 Cyg is of FUor type. V2493 Cyg was the first FUor object observed in all spectral ranges during the rise of the brightness as well as during the first year after reaching the maximum brightness. At the same time, according to existing observations the light curve of V2493 Cyg remains unique, confirming the hypothesis that each known FUor has a different rate of increase and decrease in brightness, and different light curve shape. We plan to continue our spectroscopic and photometric monitoring of the star during the next few months and years and strongly encourage similar follow-up observations.

Acknowledgements. This work was partly supported by grants DO 02-85, DO 02-273, DO-02-275, and DO 02-362 of the National Science Fund of the Ministry of Education, Youth and Science, Bulgaria. The authors thank the Director of Skinakas Observatory Prof. I. Papamastorakis and Prof. I. Papadakis for the award of telescope time and to Ivana Poljančić for the assistance in measuring Asiago plates. We also thanks Derlef Groote for providing access to the Hamburg observatory digitized plate collection. The Digitized Sky Survey was produced at the Space Telescope Science Institute under US Government grant NAG W-2166. The images of these surveys are based on photographic data obtained using the Oschin Schmidt Telescope on Palomar Mountain and the UK Schmidt Telescope. The plates were processed into the present compressed digital form with the permission of these institutions. This research has made use of the NASA Astrophysics Data System.

\section{References}

Armond, T., Reipurth, B., Bally, J., \& Aspin, C. 2011, A\&A, 528, A125 Aspin, C. 2011, AJ, 142, 135

Aspin, C., Reipurth, B., Herczeg, G. J., \& Capak, P. 2010, ApJ, 719, L50

Clarke, C., Lodato, G., Melnikov, S. Y., \& Ibrahimov, M. A. 2005, MNRAS, 361,942

Cohen, M., \& Kuhi, L. V. 1979, ApJS, 41, 743

Green, J. D., Evans, N. J., II, Kóspál, Á., et al. 2011, ApJ, 731, L25

Hartmann, L., \& Kenyon, S. J. 1996, ARA\&A, 34, 207

Herbig, G. H. 1977, ApJ, 217, 693

Herbig, G. H. 1989, in ESO Workshop on Low-Mass Star Formation and Pre-Main-Sequence Objects, ed. B. Reipurth (Garching: ESO), 233

Herbig, G. H. 2007, AJ, 133, 2679

Herbig, G. H. 2009, AJ, 138, 448

Herbig, G. H., Petrov, P. P., \& Duemmler, R. 2003, ApJ, 595, 384

Johnson, H. 1966, ARA\&A, 4, 193

Kóspál, Á., Ábrahám, P., Acosta-Pulido, J. A., et al. 2011, A\&A, 527, A133

Lee, J.-E., Kang, W., Lee, S.-G., et al. 2011, JKAS, 44, 39

Leoni, R., Larionov, V. M., Centrone, M., Giannini, T., \& Lorenzetti, D. 2010, ATel, 2854

Lorenzetti, D., Arkharov, A. A., Kopatskaya, E. N., Larionov, V. M., \& Giannini, T. 2011, ATel, 3165

Lorenzetti, D., Antoniucci, S., Giannini, T., et al. 2012, ApJ, 749, 188

Miller, A, A., Hillenbrand, L. A., Covey, K. R., et al. 2011, ApJ, 730, 80

Moro, D., \& Munari, U. 2000, A\&AS, 147, 361

Munari, U., Jurdana-Sepic, R., \& Moro, D. 2001, A\&A, 370, 503

Munari, U., Milani, A., Valisa, P., \& Semkov, E. 2010, ATel, 2808

Peneva, S. P., Semkov, E. H., \& Stavrev, K. Y. 2009, Ap\&SS, 323, 329

Peneva, S. P., Semkov, E. H., Munari, U., \& Birkle, K. 2010, A\&A, 515, A24

Pooley, D., \& Green, J. 2010, ATel, 3040

Reipurth, B., \& Aspin, C. 2010, in Evolution of Cosmic Objects through their Physical Activity, ed. H. A. Harutyunian, A. M. Mickaelian, \& Y. Terzian (Yerevan: Gitutyun), 19

Reipurth, B., \& Schneider, N. 2008, in Handbook of Star Forming Regions Vol. I, ASP, 36

Reipurth, B., Aspin, C., \& Herbig, G. H. 2012, ApJ, 748, L5

Semkov, E., \& Peneva, S. 2010a, ATel, 2801

Semkov, E., \& Peneva, S. 2010b, ATel, 2819

Semkov, E., \& Peneva, S. 2010c, IBVS, 5939

Semkov, E. H., Peneva, S. P., Munari, U., Milani, A., \& Valisa, P. 2010, A\&A, $523, \mathrm{~L} 3$

Semkov, E. H., Peneva, S. P., \& Dennefeld, M. 2011, BlgAJ, 15, 65

Wenzel, K. 2011, BAV Rundbrief, 60, 23 